\title{
Chapter 3 \\ Fostering Dialogues in Global Health Education: A Graduate and Undergraduate Approach
}

\author{
Kristin Sznajder, Dana Naughton, Anita Kar, Aarti Nagakar, \\ Joyce Mashamba, Linda Shuro, Sebalda Leshabari, and Fatou Diop
}

\subsection{Background}

Worldwide, there are new public health threats due to urbanization, climate change, demographic shifts, globalization, and political climates. At the same time, the global public health workforce may be ill-equipped to manage these threats. The current global health academic landscape in high income countries (HICs), has seen expansive growth in developing undergraduate and graduate learning opportunities aimed to provide short-term immersion in low and middle income countries [(LMICs) Jogerst et al. 2015; Panosian and Coates 2006].

There are many well-documented benefits to global health educational partnerships between the global north and global south and university linkages are a major avenue for these collaborations. For universities or host institutions in the global

\footnotetext{
K. Sznajder $(\square)$

Public Health Sciences, The Pennsylvania State University, Hershey, PA, USA

e-mail: ksznajde@phs.psu.edu

D. Naughton

Department of Biobehavioral Health, The Pennsylvania State University, University Park, PA, USA
}

\author{
A. Kar · A. Nagakar \\ Interdisciplinary School of Science, Savitribai Phule Pune University, \\ Pune, Maharashtra, India

\section{J. Mashamba $\cdot$ L. Shuro} \\ Health Promotion Unit, School of Public Health, University of Limpopo, \\ Polokwane, South Africa \\ S. Leshabari \\ Muhumbili University of Health and Allied Sciences, Dar es Salaam, Tanzania \\ F. Diop \\ Mbour Hospital, Mbour, Senegal
}


south, there is the opportunity to access greater resources and capacity from institutions in the global north, and further the reputations of educators in global health related curricula and future practitioners (Adams and Sosin 2016; Bozinoff et al. 2014; Crump and Sugarman 2008; McCoy et al. 2008). For institutions in the global north, engaging in partnerships can lead to an increased understanding of: diseases that may be more prevalent in the global south, diverse social determinants of health, and different care provision and challenges in low-resource settings (Adams and Sosin 2016; McCoy et al. 2008; Provenzano et al. 2010; Stys et al. 2013). Moreover, sequelae from these experiences have indicated an increased student interest to continue work in low-resource settings and public health arenas (Crump and Sugarman 2008; Gupta et al. 1999).

Criticism of short term global health experiences includes concerns regarding lack of knowledge about benefits to institutions and communities hosting northern students; lack of reciprocity with student or faculty exchanges; economic and labor burden to settings hosting northern students; concerns of student engagement beyond their training; lack of language training and sustainability concerns, among others (Aldulaimi and McCurry 2017; Ouma and Dimaras 2013). However, for participants in these programs regardless of where they fall on the north-south divide, there are opportunities to develop and foster intercultural awareness; and exchange knowledge across clinical, medical, public health and health science domains (Inglis et al. 2000).

Most learners on short term global health fieldwork experiences are from the global north and this leads to missed opportunities in partnership building, capacity development, and network building. These cross-cultural exchanges can benefit all participants through improving communication and adaptability to new situations, challenging notions of what global health is or is not, and bringing innovative ideas inspired through international experiences to domestic health efforts. Programs aiming to succeed in these goals must be founded on dialogic principles whereby program planning and curricula development evolve through conjoint discourse and iterative processes between north and south stakeholders. In this chapter, we share two examples in global health education that promote and operationalize bidirectionality in short-term global health exchanges: one conducted at the graduate level and one at the undergraduate level.

\subsection{An Approach in Graduate Education}

Innovative models in graduate global health education are necessary to address two main challenges in global health international experiences: (1) Learners from the global north often travel to international sites without a good understanding of the context on the ground. (2) Learners from the global south have often been excluded from the opportunity to travel and gain a world perspective related to health systems. 
Many opportunities for international field experiences at the graduate level are unstructured and oftentimes students may travel to new environments where they do not have a firm understanding of the local context, challenges, or history. The lack of structured programs for global health interns reflects missed opportunities for both students and partner institutions. Other than financial benefits, or leveraging opportunities of international collaboration, many partnering institutions do not develop precise goals about how the internship would benefit their institution or communities. The roles of these partner institutions may then become relegated to exhibiting a platform of community service, facilitating data collection through identification of sites/participants, providing students with translators and providing necessary managerial and hospitality inputs. Furthermore, weak or no mentoring is accompanied by the danger that students would return from their internship without any additional knowledge. In an alien cultural setting, students risk identifying only what they know, and collecting data on what they believe they know. When reported as publications or presentations, the internship experience would reinforce existing views, defeating the overarching goal of the short-term internship which is to acquire knowledge on health in a different setting. Lack of appropriate partner institution mentoring could also exacerbate global health inequities through reinforcing existing values without an appropriate understanding of the local context.

Partner institutions from the global south also lose an enormous opportunity for furthering global health dialogues. A structured program allows a dissection of the country's public health history, tracing how events in the past, including the colonial past of most nations of the global south have shaped the present health situation. Without a structured course, the achievements and the challenges of developing countries cannot be communicated to students visiting from regions with different socio-economic levels and standards of living. A structured program is imperative for a true discussion of "global" health. It would be essential for identifying new research areas, and breaking the monotony of focusing research activities specifically to issues relating to "unfinished agendas".

Program in India The Field Course on Public Health in India, and the accompanying online courses offered by the School of Health Sciences of Savitribai Phule Pune University (SPPU), emerged from such an argument. The Field Course aims to re-examine the existing "global" narrative on public health in India and the present ideas of public health achievements and capabilities. Globally, the health achievements of India are overshadowed by reports of its poor ranking among countries in terms of its health indicators. These rankings overshadow the fact that the country is in middle of an epidemiological transition and that the average health statistics for different states represent as a baffling range, from indicators similar to sub-Saharan African countries to those indicators that are similar to those of upper middle income countries. Although, this context of "nations within a nation" appears briefly in global literature, it defines the primary challenge facing public health in India.

The Field Course is designed as a rigorous 4 week course with the goal of illustrating the health status of the Indian population, health policies and interventions, 
and the delivery of public health interventions including local innovations. It also includes the impact of these interventions in terms of achievements, challenges, and the role of different factors in influencing the outcome of interventions. Underlying these goals is the message that while the science of public health is global, its practice and outcomes are shaped by the local context- the people, their socio-cultural, biological, physical, economic, and political environments, and the health policies, services and interventions.

The online course will flatten financial and time-related barriers to global health education between countries. Online training, prior to the country visit, would complement the onsite program developed for students from the global north visiting SPPU. This would help visiting students prepare physically and mentally to face a strange culture and environment. The on-site course is aimed at challenging students to dissect out the proximal and distal determinants that influence the efficiency of the health system in India. The courses also aim at stimulating a problem-solving approach among students.

We believe the success of the program was due to the formal structured curriculum, solid and constant student supervision, and the combination of field visits and classroom exercises. Activities such as field visits gave learners a real picture of the situation, kept learners engaged, and debunked any myths held about India and the public health system. Classroom exercises solidified course material and allowed for time to debrief experiences at the field visits. The program also invited three Indian students to formally attend the program. These students attended all classes, were present for all field visits, and engaged actively in the discussion and assignments. This gave all participants the opportunity to explore cultural context, people, and life in the city.

Program in the United States Pennsylvania State University's (PSU's) Global Health Exchange Program (GHEP) began with a goal to facilitate a global conversation between graduate students across countries and cultures about healthcare systems and models that provide access to care. By exposing graduate students early to diverse approaches to health care access and models of healthcare delivery systems, students gain a broader and more informed global perspective. A global perspective will benefit students throughout their career and may ultimately benefit their own local public health community through bringing a unique perspective and open mind to possible public health solutions.

GHEP invites students from international partner institutions to participate in a public health training course in Hershey, Pennsylvania and sends PSU students to international partner institutions to gain first-hand perspectives on health care challenges and systems in each host country. GHEP's structure is grounded in university linkages that offer structured international programs for PSU students and are interested in sending their students to PSU for a structured program. GHEP aims to prime learners on how to function in a new health system and how to understand the way other health systems work and why. This focus on having learners understand how to assess and approach different health systems was inspired by the 2010 Lancet Commission report that called for an approach that is 
'systems based to improve the performance of health systems by adapting core professional competencies to specific contexts, while drawing on global knowledge' (Frenk et al. 2010:1924). This two-way exchange program is based in the global health competencies set forth by the Association of Schools and Programs in Public Health and the Consortium for Universities in Global Health (Ablah et al. 2014; Jogerst et al. 2015).

PSU students are able to complete their required three credit internship through traveling abroad with GHEP. GHEP international partner institutions each offer programs to immerse students in the health system and culture in their country and offer mentored projects suitable to each learner's area of interest or skillset. Before travel, PSU students attend preparatory meetings that cover an overview of the health system and current events in the country where they will complete their internship, health and safety, and cultural humility. Students are required to keep a daily log of reflections and submit a final assignment related to their mentored project.

GHEP's short-term summer training program in the United States (US) provides the opportunity for international students to gain a first-hand look into the US healthcare system. The intensive course includes faculty lectures on US governmental structure and health policy, as well as discussions of current major public health challenges faced by the US. These knowledge-based sessions are supported through site visits to national and state government offices, as well as community organizations working to improve health and advocate for change on a local level. Concurrently, students visit different types of healthcare facilities to gain a better understanding of the levels and options for care in the US. PSU students are also offered the opportunity to attend this course to foster student dialogue in health and make connections for students outside of the US. At the end of the program, students are asked to conduct a presentation in pairs that join students from different countries and compare health challenges and systems in their home countries and the US. In the end, students should come away with a good understanding of the US healthcare system and how it compares across the countries in which we have student representation.

\subsection{An Approach in Undergraduate Education}

PSU's global health education efforts at the undergraduate level aim to facilitate the expansion of students' interests in global health care provision, access and issues through the offering of a 21-27 credit minor in global health. This program is transdisciplinary - open to majors across the university, and exposes students to theoretical, scientific and practical issues affecting the health of people in various countries and world regions. Comprised of both an academic in-class component and an experiential six-week fieldwork segment, it typically attracts students envisioning careers in medicine, public health, or work in government and non-profit sectors. Through a series of courses students gain understanding of a range of issues that cut across global health domains and competencies promulgated by the Consortium of 
Universities for Global Health (CUGH) (Jogerst et al. 2015). These include building knowledge, skills and attitudes across key domains including burden of disease; health and globalization; health determinants; capacity strengthening; promoting collaboration and partnering; ethics; professional practice; and health equity and social justice (Jogerst et al. 2015). In addition, students take a pre-fieldwork course focusing on the culture, health systems and current issues in their fieldwork region; critiques and issues of short-term global health assignments; and psychosocial areas of preparation for this form of immersion experience. Students typically enter the program with little to no clinical care experience. From both an ethical and administrative stance, as well as considerations of risk management, students are taught to understand that their role during the field work segment abroad is as student learners. Within the realm of medical activities, they are observers who shadow and accompany foreign medical professionals and medical students as they undertake hospital and clinic rotations in rural and urban settings.

The field sites in South Africa, Tanzania, and Senegal are forged through official partnerships between PSU and departments in nursing, public health, medicine and other related health disciplines at our partner universities. Formal and transparent relationships between the university and host partners are established through multiyear memoranda of understanding and agreements (MOUs and MOAs). These documents along with consistent email and telephone contact throughout the year, help form partnerships that work together to coordinate student and program logistics from faculty payment to students' short term enrollments in partnering African universities and obtain permissions for student rotations in health and community settings.

An additional level of engagement, targeted to minimize unidirectionality of agency between U.S. and host institutions (Loh et al. 2015) is an annual or bi-annual planning workshop. Sponsored by PSU with costs covered by the program, the weeklong event invites host country faculty coordinators to participate in fieldwork planning, design, implementation and evaluation. With 15-25 PSU students studying across three countries, it is imperative that host partners' perspectives and capacities are understood and privileged. Workshop topics include specific planning and administration reviews, program evaluation and areas of improvement, risk management issues, pedagogical approaches, field site considerations (for example extending or contracting clinical or public health focused rotations), changes in policies, and other areas. Prior to attending, host faculty members are invited to offer academic presentations of their research or work to our university audiences and arrangements are typically made for videoconferencing of presentations.

Program Design and the South African-USA Program As university educators, administrators and healthcare professionals in the field site in South Africa, the participatory and interactive undergraduate program model is an important model to facilitate experiences in global health. At the South African (SA) University, mentors are involved in developing a program schedule determined by areas of interests of the participating students, the visit by the African faculty to USA and most importantly, the local context. The program schedule in the host country is designed 
to expose students to various aspects of the South African health care system at both health care settings and within the community.

During the 6 week field experience, students are scheduled to participate and interact in the following settings:

1. Hospital/Clinic setting: In this setting, students, students are involved in shadowing health professionals such as doctors and community outreach workers and are exposed to health policy and management. The students gain knowledge and skills as they are exposed to the extent of the burden of disease in SA as well health care practices within the settings.

2. Community setting: In this setting students are exposed to community- based health care workers, old age groups tackling chronic diseases, youth friendly health centers dealing with reproductive health, the private hospital structure, nutrition within private clinics, health-promoting schools, and community projects such as greenery projects. This experience highlights to students the health inequalities, the risk factors, health needs and responses thereof within the society.

3. School Needs Assessment and Project: Toward the end of the field visit, the US students undertake a project in schools (middle to high school levels) in which they conduct a health talk and design health promotion material for learners. The project is based on a needs assessment that they conduct in which they plan and address priority health issues of the school students. Through the project, the undergraduates learn the skills of conducting a needs assessment before designing a health promotion project and health talks based on priority of issues. The program helps to exchange information throughout the 6 weeks and at the end students present their experiences as a presentation to our university faculty, administrators and their medical mentors in the health communities.

In short, the model facilitates the students to gain knowledge and skills on the various aspects of global health (burden of diseases, health care practices, research, health policy and management, primary health care and determinants of health in SA). Some cultural aspects and beliefs within South Africa are explored as these contribute significantly to the health of the people.

For the US program planning component, South African university management provides permission for the faculty to visit the USA based on the Memorandum of understanding (MOU) and the Memorandum of Agreement (MOA). The plans are made well in advance of the proposed period of the activity. Orientation is planned and prepared for participating students at the initial part of the program at the host country. Prior to placement of students to various settings, in-country orientation is conducted for the students around issues of security, transportation, accommodation, social life, the university and surrounding communities. Students are also introduced to the University management and key staff members. This allows students to know the country, be aware of existing health needs and different ways of responding to such needs. The plans are made flexible to accommodate participating student's quest for knowledge and people they would like to meet during the period of their stay. 
Program Design and the Senegal-USA Program Perspective The internship in Mbour, Senegal is a great opportunity for students and for the hospital. Indeed, the students find themselves in a context that puts him to the test with the discovery of new global health realities; it requires an open mind and a capacity for adaptation. The field work experience should allow students to gain maturity. The hospital is open to global partnerships and to the benefits derived from networking with other partners. An inter-hospital twinning with Mbour Hospital and a global US partner would be an asset to our development.

The Senegal program culture-centric approach to training students, and developing community-academic partnerships. Daily, students rotate through the main hospital in MBour Senegal, participate in research projects with supervising US and Senegalese faculty and attend local research conferences and events.

The goal is to have students engage in the local culture as a vehicle for understanding local health. To achieve this goal we employ two major strategies. First we partner with a nonprofit organization with sites in the US and Senegal that takes a grassroots, community-based approach to non-communicable disease prevention and management. This organization, allows students to participate in local community health screenings and community health capacity building programming such as diabetes support group meetings and events while in Senegal.

Second, we engage in cultural activities on a weekly basis. The premise of the Senegal site is to learn about health through culture. Students attend athletic events, music events, are hosted for dinner by local families, take Senegalese dance class, attend weddings, visit cultural sites such as Goree Island, shop at local markets and learn the local language. Program leaders from both Senegal and the US have daily discussions with students about how they are interpreting local culture, context and health issues. While ultimately the cultural emersion helps the students gain, students may feel awkward or uncomfortable being an outsider and often need help to process daily experiences.

African Faculty Program Planning USA Meeting Perspective Meeting with the coordinators from the other African countries along with PSU program administrators and faculty provided a critical and important opportunity of discussing face-toface issues pertaining to the program - the strengths, challenges and further improvements including sustainability. We learn many things from each other. This creates a sense of friendship and ownership of the program. It creates a friendly networking South - South and also South - North kind of close relationship. Automatically, we feel valued and our confidence and motivation in coordinating this program is enhanced again and again.

Conducting in person PSU is an opportune time to familiarize ourselves with the students and make friends with them when they come to the country. It gives them knowledge of our local culture and customs before arriving in the host country. It creates a unique relationship, not as new people when we meet in the country. Their conversation and questions mostly serves to help us know their expectations and make preparations accordingly. It helps allay culture shock and prepares students' adjustment to the foreign culture. 
Inter-program exchanges can lead to other benefits such as learning new skills and techniques. Visiting PSU also helps improve my teaching skills and ability to handle students. In addition, it makes the coordinators familiar with resources such as how to teach in the simulation lab. For example, the US simulation lab is of a high standard and from our time visiting it, I learned how to improve our simulation lab in Muhumbili, Tanzania. Future improvements to the USA-African program could benefit from online sessions between Pennsylvania State University and our universities using video - conferencing where the students from each side can learn from each other and familiarize prior to onsite meeting in the countries.

\subsection{Conclusion}

Efforts in bilateral partnerships in both graduate and undergraduate global health educational programs offer multiple benefits for involved institutions. The benefits reported in the literature include the opportunity to strengthen ethical partnerships, educate the global workforce, empower trainees to be agents of change in their home institutions, and improve training and opportunities at host institutions (Arora et al. 2017). In addition to those advantages, our bidirectional global health programs yield the following rewards including increased interaction between faculty and students in the global north and the global south, strengthened sense of community between institutions, and an improved understanding of diverse health care systems, approaches to health challenges, and cultural values related to health and health care delivery.

Challenges related to our bidirectional global health education programs include funding students and faculty from the global south to travel to the United States for training and creating structured programs that minimize the burden of hosting institutions including orienting students to the local context, logistics, curriculum development, cultural or language barriers, and mentorship (Arora et al. 2017). The time required to host students and ensure a successful visit can be disruptive and burdensome for faculty and staff at host institutions and take away time from regular commitments (Provenzano et al. 2010). Before any educational partnership is established, it is necessary to weigh the benefits of the exchange with the costs of diversion of faculty and staff time (Provenzano et al. 2010). It is also important to communicate with host institutions to establish their needs to run such a program. Additionally, it may be difficult to find ways for students to benefit the host institution and serve the needs of the community in which they visit (Provenzano et al. 2010). Structured and reciprocal educational programs strive to meet these challenges, but can fall short. It can be difficult to identify areas for reciprocal arrangements due to challenges in aligning faculty priorities in terms of the proportion of effort dedicated to teaching and research, educational program goals including necessary competencies and goals students are required to meet through international experiences, as well as institutional priorities in terms of differing university strategic goals and resources available that support educational initiatives. These challenges are difficult to overcome and each institution will have their own approaches to mitigating the challenges. Facilitating early and often communication is critical to finding 
appropriate solutions to financial, logistical, educational, cultural/language, and mentorship concerns.

The educational programs developed by the SPPU and PSU helped build bilateral relationships between institutions and faculty, educate students about the public health systems in India and the United States, incorporate views of the local experts, and expose students to various dimensions of health challenges in India and the United States. The undergraduate global health educational program through PSU and its partners in Africa offers a model for bidirectional faculty collaboration. Both models for global health education can provide new ideas for global health educational development and partnership.

This chapter describes two distinct methods of strengthening global health educational partnerships and increasing bilateral dialogue between partners at the student and faculty level. Short-term international internships allow for a unique opportunity to support bilateral partnerships in global health education. Further expansion of bilateral global health educational programs is necessary for strengthening international partnerships and building a global health workforce that has a shared experience in global health exchange and bidirectional dialogue.

\section{Challenges}

- Identifying opportunities for mutual benefit between institutions is difficult due to potential misalignment in faculty priorities, required competencies for students, as well as possible diverging institutional priorities.

- Students need to be prepared with a good understanding of the local context surrounding public health challenges and initiatives before they travel for international global health opportunities.

- Adequate resources need to be allocated to students and faculty from the global south to participate in domestic and international global health trainings and collaborative meetings

\section{Lessons Learned}

- Short term international internships allow for a unique opportunity to support bilateral partnerships in global health education.

- Further expansion of bilateral global health educational programs is necessary for strengthening international partnerships and building a global health workforce that has a shared experience in global health exchange and bidirectional dialogue.

\section{References}

Ablah E, Biberman DA, Weist EM et al (2014) Improving global health education: development of a global health competency model. Am J Trop Med Hyg 90(3):560-565. https://doi. org/10.4269/ajtmh.13-0537

Adams LV, Sosin AN (2016) Beyond visas and vaccines: preparing students for domestic and global health engagement. Ann Glob Health 82(6):1056-1063. https://doi.org/10.1016/j. aogh.2016.10.010 
Aldulaimi S, McCurry V (2017) Ethical considerations when sending medical trainees abroad for global health experiences. Ann Glob Health 83(2):356-358. https://doi.org/10.1016/j. aogh.2017.03.001

Arora G, Russ C, Batra M et al (2017) Bidirectional exchange in global health: moving toward true global health partnership. Am J Trop Med Hyg 97(1):6-9. https://doi.org/10.4269/ ajtmh.16-0982

Bozinoff N, Dorman KP, Kerr D et al (2014) Toward reciprocity: host supervisor perspectives on international medical electives. Med Educ 48(4):397-404. https://doi.org/10.1111/medu.12386

Crump JA, Sugarman J (2008) Ethical considerations for short-term experiences by trainees in global health. JAMA 300(12):1456-1458. https://doi.org/10.1001/jama.300.12.1456

Frenk J, Chen L, Bhutta ZA et al (2010) Health professionals for a new century: transforming education to strengthen health systems in an interdependent world. Lancet 376(9756):1923-1958. https://doi.org/10.1016/S0140-6736(10)61854-5

Gupta AR, Wells CK, Horwitz RI et al (1999) The International Health Program: the fifteen-year experience with Yale University's Internal Medicine Residency Program. Am J Trop Med Hyg 61(6):1019-1023

Inglis A, Rolls C, Kristy S (2000) The impact on attitudes towards cultural difference of participation in a health focused study abroad program. Contemp Nurse 9(3-4):246-255

Jogerst K, Callender B, Adams V et al (2015) Identifying interprofessional global health competencies for 21st-century health professionals. Ann Glob Health 81(2):239-247. https://doi. org/10.1016/j.aogh.2015.03.006

Loh LC, Cherniak W, Dreifuss BA et al (2015) Short term global health experiences and local partnership models: a framework. Glob Health 11(1):50. https://doi.org/10.1186/ s12992-015-0135-7

McCoy D, Mwansambo C, Costello A et al (2008) Academic partnerships between rich and poor countries. Lancet 371(9618):1055-1057. https://doi.org/10.1016/S0140-6736(08)60466-3

Ouma BD, Dimaras H (2013) Views from the global south: exploring how student volunteers from the global north can achieve sustainable impact in global health. Glob Health 9(1):32. https:// doi.org/10.1186/1744-8603-9-32

Panosian C, Coates TJ (2006) The new medical "missionaries"- grooming the next generation of global health workers. N Engl J Med 354(17):1771-1773. https://doi.org/10.1056/ NEJMp068035

Provenzano AM, Graber LK, Elansary M et al (2010) Short-term global health research projects by US medical students: ethical challenges for partnerships. Am J Trop Med Hyg 83(2):211-214. https://doi.org/10.4269/ajtmh.2010.09-0692

Stys D, Hopman W, Carpenter J (2013) What is the value of global health electives during medical school? Med Teach 35(3):209-218. https://doi.org/10.3109/0142159X.2012.731107

Open Access This chapter is licensed under the terms of the Creative Commons Attribution 4.0 International License (http://creativecommons.org/licenses/by/4.0/), which permits use, sharing, adaptation, distribution and reproduction in any medium or format, as long as you give appropriate credit to the original author(s) and the source, provide a link to the Creative Commons license and indicate if changes were made.

The images or other third party material in this chapter are included in the chapter's Creative Commons license, unless indicated otherwise in a credit line to the material. If material is not included in the chapter's Creative Commons license and your intended use is not permitted by statutory regulation or exceeds the permitted use, you will need to obtain permission directly from the copyright holder.

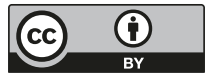

